\title{
BMJ Open Effectiveness of breathing exercises, foot reflexology and back massage (BRM) on labour pain, anxiety, duration, satisfaction, stress hormones and newborn outcomes among primigravidae during the first stage of labour in Saudi Arabia: a study protocol for a randomised controlled trial
}

Kamilya Jamel Baljon (D , , ${ }^{1,2}$ Muhammad Hibatullah Romli, ${ }^{3}$ Adibah Hanim Ismail, ${ }^{1}$ Lee Khuan, ${ }^{3}$ Boon How Chew (i) ${ }^{1}$

To cite: Baljon KJ, Romli MH, Ismail AH, et al. Effectiveness of breathing exercises, foot reflexology and back massage (BRM) on labour pain, anxiety, duration, satisfaction, stress hormones and newborn outcomes among primigravidae during the first stage of labour in Saudi Arabia: a study protocol for a randomised controlled trial. BMJ Open 2020;10:e033844. doi:10.1136/ bmjopen-2019-033844

- Prepublication history for this paper is available online. To view these files, please visit the journal online (http://dx.doi. org/10.1136/bmjopen-2019033844).

Received 24 August 2019

Revised 14 April 2020

Accepted 20 May 2020

Check for updates

(C) Author(s) (or their employer(s)) 2020. Re-use permitted under CC BY-NC. No commercial re-use. See rights and permissions. Published by BMJ.

For numbered affiliations see end of article.

Correspondence to Dr Boon How Chew; chewboonhow@upm.edu.my

\section{ABSTRACT}

Introduction Labour pain is among the severest pains primigravidae may experience during pregnancy. Failure to address labour pain and anxiety may lead to abnormal labour. Despite the many complementary nonpharmacological approaches to coping with labour pain, the quality of evidence is low and best approaches are not established. This study protocol describes a proposed investigation of the effects of a combination of breathing exercises, foot reflexology and back massage (BRM) on the labour experiences of primigravidae.

Methods and analysis This randomised controlled trial will involve an intervention group receiving BRM and standard labour care, and a control group receiving only standard labour care. Primigravidae of 26-34 weeks of gestation without chronic diseases or pregnancyrelated complications will be recruited from antenatal clinics. Eligible and consenting patients will be randomly allocated to the intervention or the control group stratified by intramuscular pethidine use. The BRM intervention will be delivered by a trained massage therapist. The primary outcomes of labour pain and anxiety will be measured during and after uterine contractions at baseline (cervical dilatation $6 \mathrm{~cm}$ ) and post BRM hourly for 2 hours. The secondary outcomes include maternal stress hormone (adrenocorticotropic hormone, cortisol and oxytocin) levels, maternal vital signs (V/S), fetal heart rate, labour duration, Apgar scores and maternal satisfaction. The sample size is estimated based on the between-group difference of 0.6 in anxiety scores, $95 \%$ power and $5 \%$ $\alpha$ error, which yields a required sample size of 154 (77 in each group) accounting for a $20 \%$ attrition rate. The between-group and within-group outcome measures will be examined with mixed-effect regression models, time series analyses and paired t-test or equivalent nonparametric tests, respectively.

\section{Strengths and limitations of this study}

This single-blind, parallel, randomised controlled trial will explore the combined effects of breathing exercises, foot reflexology and back massage (BRM) on pain and anxiety during labour in healthy primigravidae with a singleton fetus.

- The effects of BRM will also be examined through objective physiological measurement of stress hormone levels and comparison of these levels between groups before and after the intervention.

- The intervention will be applied for 1 hour and only once during the first stage of labour after cervical dilatation of $6 \mathrm{~cm}$.

- Blinding of the primigravidae mothers is not possible, and there may be bias in the self-assessed subjective outcomes such as the Visual Analogue Scale.

- The expertise and experience of the nursing graduates who are trained to be the massage therapists is considered an important factor in the quality of treatment provided and this may underestimate the effect of BRM.

Ethics and dissemination Ethical approval was obtained from the Ethical Committee for Research Involving Human Subjects of the Ministry of Health in the Saudi Arabia (H-02-K-076-0319-109) on 14 April 2019, and from the Ethics Committee for Research Involving Human Subjects (JKEUPM) Universiti Putra Malaysia on 23 October 2019, reference number: JKEUPM-2019-169. Written informed consent will be obtained from all participants. Results from this trial will be presented at regional, national and international conferences and published in indexed journals. Trial registration number ISRCTN87414969, registered 3 May 2019. 


\section{INTRODUCTION}

Many primigravidae have reported experiencing various levels of pain during labour and high levels of anxiety about the labour process and its outcomes. ${ }^{1-3}$ Anxiety escalating to fear is a common issue related to labour, especially among primigravidae. ${ }^{45}$ Other recorded negative perceptions and psychological effects influencing labour experiences include distress and feelings of powerlessness during labour for women and their families. ${ }^{5-7}$

When poorly managed, labour pain may lead to severe consequences for women, such as prolonged labour, ${ }^{58}$ which may increase the risk of fetal distress, head compression, intrauterine fetal death, low Apgar scores and physical injuries to neonates. ${ }^{59}$ Prolonged labour results in increased risk of caesarean section, induced labour and assisted delivery using vacuum and forceps. ${ }^{10}{ }^{11}$ Studies have also reported negative mental impacts on women, sometimes even including postnatal post-traumatic stress disorder, ${ }^{12}{ }^{13}$ and subsequently reduced quality of life. ${ }^{14}$ Feelings of anxiety often originate from possible birthing complications about which pregnant women have heard and read, ${ }^{451516}$ and may even result in women refusing normal vaginal delivery and insisting on caesarean sections without medical indications. ${ }^{17}$ It is therefore important for healthcare professionals to assist and educate all expectant mothers on labour pain management.

Appropriate labour pain management and interventions are important aspects of obstetric care to ensure optimum outcomes for mothers and babies. ${ }^{18}$ Pharmacological interventions used in the management of labour pain include systemic sedatives, analgesics and regional anaesthesia. ${ }^{19}$ Examples of these analgesics are aerosol and epidural opioids, intramuscular pethidine (IMP) and intravenous sedatives. ${ }^{20}{ }^{21}$ Some of these are expensive and may be associated with adverse effects on mothers, the labour process and neonates. ${ }^{22}$ In contrast, most nonpharmacological methods for labour pain management are simple and non-invasive, and are often cheaper and safer than pharmacological interventions. ${ }^{23-25}$ Studies have found that non-pharmacological approaches, particularly breathing exercises, have positive impacts on relief of labour pain, ${ }^{26-28}$ and anxiety in pregnant mothers. ${ }^{29-31}$ This is especially true for Lamaze breathing, deep breathing exercises, ${ }^{26-2832} 33$ reflexology ${ }^{634}$ and massage. ${ }^{35}$ Non-pharmacological approaches have been linked to shorter labour duration, ${ }^{36}$ and improved newborn outcomes. ${ }^{37}$ Our systematic review found that massage is beneficial for relieving labour pain, ${ }^{38}$ and is associated with greater relaxation, higher alertness levels, improved mood and reduced stress hormone (cortisol) levels and anxiety symptoms. ${ }^{39}$

\section{Rationale}

It is hypothesised that the non-pharmacological approach of labour pain management occurs via the alteration of nociceptive stimuli and modification of the processing of nociceptive input at the central level, resulting in an overall improved sense of comfort and well-being, ultimately leading to stronger coping capabilities by the mothers in labour. ${ }^{40}$

The physiological mechanism of breathing is a protective action as it is a fight-or-flight reflex triggered by the central nervous system. Physiologically, deep abdominal breathing stimulates the parasympathetic nervous system. As a result, the blood circulation in pregnant women will undergo oxygenation, which will trigger the release of endorphins associated with decrease in heart rate and increase in feelings of calmness. At the same time, endorphins can also suppress the sympathetic system, leading to a decrease in the release of stress hormones such as cortisol. $^{4142}$

As for reflexology, so far there has been no constructive explanation of the underlying mechanism in reducing labour pain. ${ }^{636}$ The reflexology therapist will apply pressure three times on specific points of the feet that are energetically connected to certain parts and organs of the body. As with skin-to-skin contact during massage, reflexology point pressure could trigger the release of endogenous endorphins and encephalins that help to reduce labour pain, stress, fatigue and anxiety. ${ }^{43-46}$ Pressure on the solar plexus at the border of the upper and middle one-third of the sole is believed to facilitate the functions of the body's nervous system. ${ }^{47}$ Pressure on the lower part of the forefoot reflects the heart and lungs. While pressure on the bridge of the foot reflects the liver and kidney, the heel will reflect the lower back, legs, pelvic region uterus and intestines. The uterine point is believed to be located in the indented region between the inner ankles and the sole. ${ }^{48}$ Therefore, it is believed to be helpful during labour. The pressure on toe and heel stimulate the reflex points in the pelvis. It is effective by releasing the oxytocin hormone which start and regulate the uterine contractions and relax during contractions. ${ }^{49}$

However, there are several postulated theories for its mechanism of action. First, the autonomic-somatic integration theory suggests that the pressure applied to the feet during reflexology compresses the receptors in the cells, thus opening up the ionic channels in the plasma membrane and triggering a local action with the potential to convey messages to the spinal cord and/or brain. ${ }^{46}$ The application of alternating pressure to the feet may also produce predictable reflexive actions within the nervous system and activate the parasympathetic nervous system. ${ }^{50}$ Based on the energy theory that posits that energy moves towards the head from reflex points that stimulate the neural paths, improve blood flow, release the endorphins and relief pain. ${ }^{51}$ Another contemporary method explains that reflexology acts through 'sympathetic resonance', in which an energy wave flows between therapist and client, promoting homeostatic balance ${ }^{52}$ This may occur through local enzymatic reactions on receptive fields or through an improved blood supply as a result of local skin temperature changes following the skin-to-skin contact. ${ }^{47}$ Reflexologists also believe that the application of deep pressure on certain reflex points of the sole and palm may break any calcium crystals and uric 


\section{Mechanisms of the Reflexology}

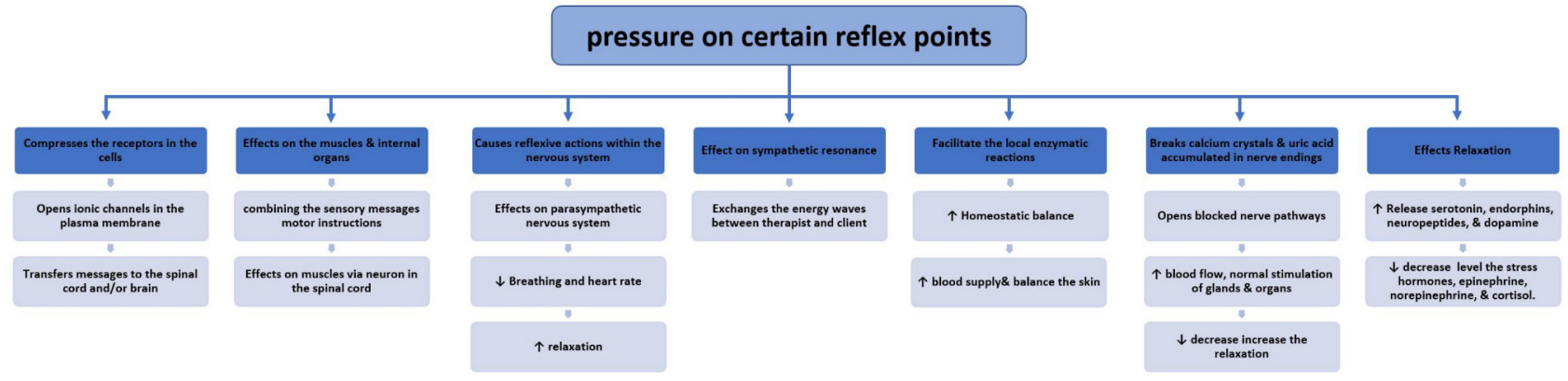

Figure 1 Mechanisms of action reflexology therapy.

acid accumulated in nerve endings that may cause blockages and induce pain. ${ }^{53}$

Reflexology also results in body relaxation and stimulation of any blocked nerve endings, which may propel any sluggish glands or organs to regain their normal functioning. ${ }^{54}$ Ambiguity remains regarding the theories and mechanism of action of foot reflexology for labour pain, as compared with that for general pain. ${ }^{6356}$ Nonetheless, it is plausible to believe that reflexology techniques would have similar physiological effects for labour pain that bring about a sense of well-being, analgesia and subsequently the perception of pain relief. ${ }^{37}$ Figure 1 summarises the possible mechanisms of reflexology therapy.

Massage therapy is another type of commonly used complementary and alternative medicine (CAM) for the promotion of health and well-being. ${ }^{35}$ Massage is a potent mechanical stimulus that produces a short-lived analgesic effect by activating the 'pain gate' mechanism. ${ }^{55}$ Longer lasting pain control appears to be mediated mainly by the descending pain suppression mechanism by activation of descending efferent pathways. ${ }^{56}$ The inhibition of pain-transmission neurons involves a combination of physiological and neurological mechanisms and it is commonly activated by noxious stimulation. ${ }^{57}$ Figure 2 summarises the possible mechanisms action of massage therapy. $^{58}$

The three aforementioned therapies (ie, breathing exercises, foot reflexology and backmassage (BRM)) for labour pain management have been shown to influence the secretion of certain stress hormones such as cortisol, adrenocorticotropic hormone (ACTH) 3959 oxytocin (OT), ${ }^{59}$ and possibly also the endorphins. ${ }^{44} 45$ Endogenous oxytocin is a key component in the molecular pathways that buffer reaction to stress and decrease sensitivity to pain and inflammation ${ }^{60}$; cortisol is an important hormone released during stressful conditions. $^{39}$

Figure 2 Mechanisms of action massage therapy. 


\section{Significance of this clinical trial}

Many studies have reported that not all pharmacological and non-pharmacological methods on their own are able to reduce labour pain satisfactorily. Despite the intervention, some mothers still endure some pain, anxiety and prolonged labour, and suffer from negative maternal and perinatal consequences. ${ }^{5}{ }^{516}$ From the perspective of complementary management, BRM are the techniques with the highest potential for managing pain and anxiety for primigravidae. Systematic reviews have concluded that CAM interventions to manage pain and anxiety during labour have often been biased and/or poorly executed, thus resulting in low quality of evidence, ${ }^{61-64}$ or no strongly supported evidence. ${ }^{65-67}$ Therefore, there is a need for a rigorous and robust trial to examine the effect of the combined intervention of BRM on labour pain, anxiety, stress hormones, vital signs $(\mathrm{V} / \mathrm{S})$, fetal heart rate (FHR), duration of labour, Apgar scores and maternal satisfaction among the primigravidae using multiple relevant outcome measures.

\section{METHODS AND DESIGN}

This study aims to investigate the combined effect of BRM on labour pain, duration of labour, anxiety, maternal satisfaction, stress hormones and newborn outcome among primigravidae in Saudi Arabia. The specific objectives are (1) to compare the effect of the combined breathing exercise, foot reflexology and back massage (intervention) on labour pain intensity, anxiety level, duration of labour, maternal satisfaction, stress hormones and neonatal outcome compared with the standard midwifery care (control); (2) to identify the predictors of pain, anxiety, duration of labour, the satisfaction of mother and neonatal outcome from the baseline sociodemographic and obstetric characteristics.

\section{Study design}

The study design will be a single-blind parallel randomised controlled trial (RCT), in which participants are randomly assigned to receive either the BRM intervention or control care.

\section{Study setting}

This study will be conducted in the Makkah Maternity and Children Hospital (MCH) in Makkah, Saudi Arabia. The hospital is a tertiary-level, governmental referral hospital with special services for paediatrics, gynaecology and obstetrics. ${ }^{68}$ In Saudi Arabia, almost all tertiary hospitals, including our study site, offer systemic pharmacological agents, either intravenous or intramuscular analgesics to manage pain during labour ${ }^{69}$; however, providing non-invasive and non-pharmacological methods of pain relief during labour is not common practices. ${ }^{69}$ To our best knowledge, the combined effect of BRM on primigravidae has not been investigated at any Saudi Arabia hospital prior to this trial.

\section{Participants}

The study participants will include primigravidae, age 20-35 years old, at 37-41 weeks of gestation, and in the first stage of labour. The inclusion criteria include singleton pregnancy, cephalic presentation and regular contraction. In labour, the participants must achieve $6 \mathrm{~cm}$ of cervical dilatation, with a minimum of three contractions of at least moderate intensity every $10 \mathrm{~min}$, in which the duration of the contraction must be between $30 \mathrm{~s}$ and $60 \mathrm{~s}$.

The exclusion criteria include diagnosis of underlying chronic diseases such as cardiovascular disease, kidney disease, diabetes, asthma, mental health disorders, epilepsy or seizure; pregnancy-related diseases such as gestational diabetes, preeclampsia, cephalo-pelvic disproportion, polyhydramnios or oligohydramnios or deep venous thrombosis; and pregnancy complications such as placenta praevia, antepartum haemorrhage, fetal distress or being put on analgesics other than IMP.

\section{Recruitment}

Recruitment will be conducted at the antenatal clinic at the trial site. Only those who plan to deliver in the trial hospital's delivery room will be further briefed and assessed for their eligibility. At this hospital, antenatal mothers are given monthly follow-up appointments until 28 weeks' gestation. The frequency increases to biweekly until 32 weeks' gestation; then patients are seen weekly until delivery.

For this study, we will approach primigravidae between 26 weeks and 34 weeks of gestation in equal numbers based on the gestational weeks. This means that about an equal number of primigravidae at week of gestation of 26 , $28,30,32$ and 34 will be recruited in order to spread out the occurrence of labour in the subsequent 2-3 months to increase the feasibility of the BRM intervention. Because participant recruitment and the training of the research team members are estimated to last up to 2-3 months, women of $34+$ weeks gestation cannot be recruited during this period because they will inevitably go into labour before the research preparations are complete.

At the antenatal clinic, the principal investigator will provide general health education about pain management during labour. The participant information sheet of this RCT will be provided for the eligible patients. If they are interested in participating, they will sign a written consent form and will be identified by a unique stamp on their antenatal cards. When the participants arrive in the labour room for delivery, they will be re-evaluated for the eligibility.

\section{Randomisation}

Since IMP is a commonly prescribed analgesic in labour and may have substantial effects on the primigravidae and neonates, randomisation will be stratified according to the administrative status of IMP. This will ensure the same numbers of primigravidae with and without IMP in the intervention and control groups. To achieve this, we 


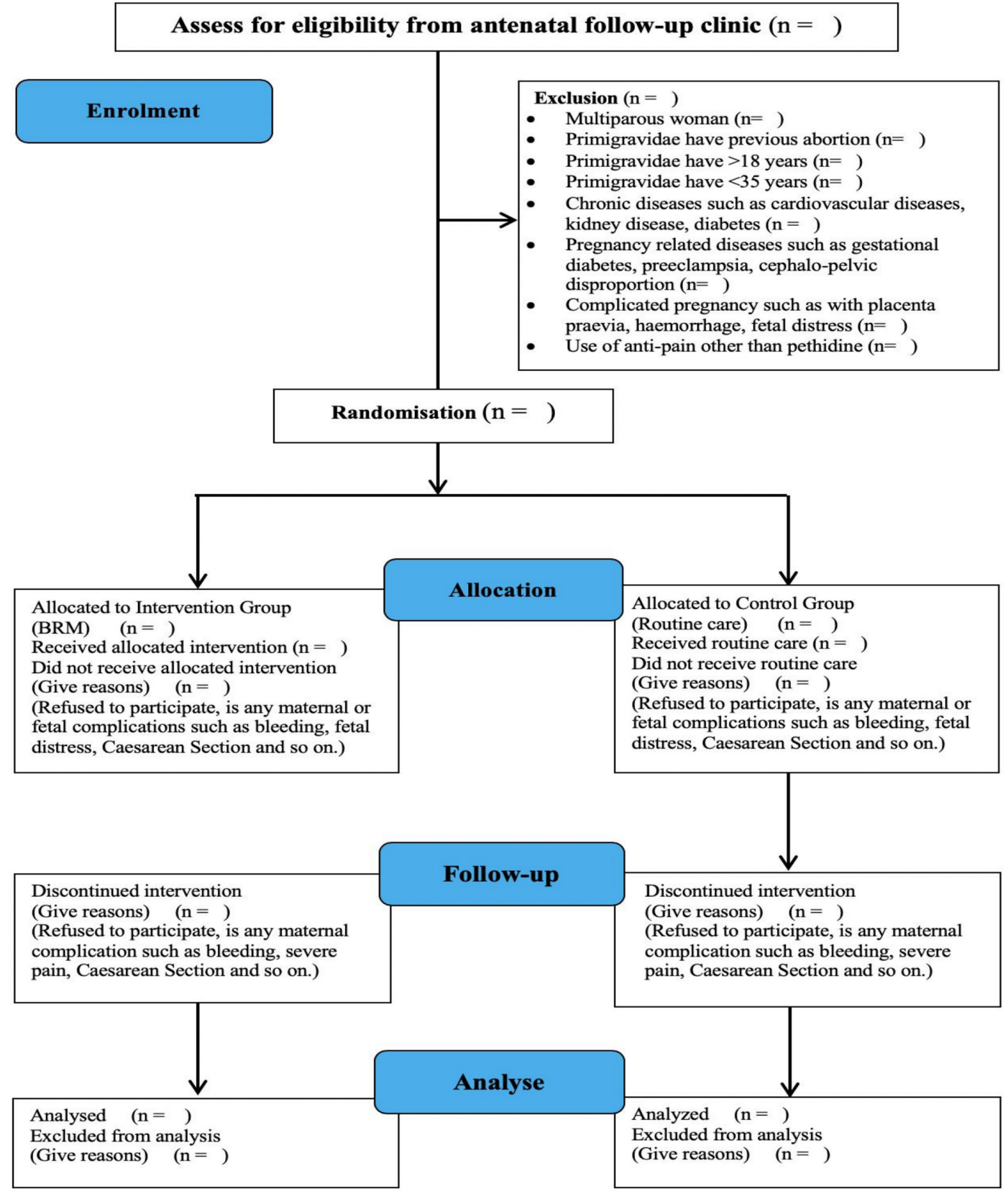

Figure 3 Consolidated Standards of Reporting Trials flow diagram.

use a block of size 4 with a 1:1 allocation ratio, leading to a possibility of 6 permutations. All possible block sequences will be randomly generated with the help of free software from the internet https://www.sealedenvelope.com/ simple-randomiser/v1/. A random list will be created after the sample size number, treatment groups, block sizes, list length and stratification factors are entered into the software. The order of the subjects will be used by the research coordinator who will be stationed in the delivery room to conduct the random group allocation for primigravidae in labour who have achieved a cervical dilation of $6 \mathrm{~cm}$. The principal investigator, outcome assessors and massage therapist in this trial will not be involved in the allocation of the interventional groups. Figure 3 outlines the Consolidated Standards of Reporting Trials flow diagram.

\section{Data collection}

Every questionnaire will be coded with a unique number. Data collection in the delivery room will be facilitated by the trained research coordinator and two outcome assessors. The outcome assessors will be assigned to the control or the intervention group on the same day. Once the form is completed by the outcome assessors, it will be kept by the research coordinator in a safe location in the delivery room.

Throughout all of the outcome assessment time points, a massage therapist will be present in the delivery room of 
both the intervention and control groups. For the intervention group, the primigravidae in labour will receive the BRM intervention from the massage therapist. For the control group, the practicing midwife will perform routine labour care such as touch therapy, ensuring that the mother lies on her left side, and providing encouragement and counselling. The outcome assessor will measure and assess both the intervention and control groups at the same time points. Both groups will be equipped with similar extra equipment. This blinding effort is intended to minimise biases during the outcome assessment. However, blinding of the participants will be impossible due to the nature of the intervention.

\section{Interventions}

The BRM intervention consists of 5 min of breathing exercise followed by $10 \mathrm{~min}$ of foot reflexology on each sole and $35 \mathrm{~min}$ of continuous massage over the lower limbs and back. The massage therapist will allow the primigravidae to lie on the left side,$^{70}$ to move and change her position during the intervention and answer any question or inquiry. Table 1 provides a detailed explanation of the BRM intervention procedure. As for the control group, the primigravidae in labour will receive routine labour room care.

\section{Training for the research team members}

A total of 13 research assistants will be recruited and trained for the intervention and data collection from June to December 2019, (figure 4). They will be given the BRM training for 1 week by the principal investigator who has completed the professional massage and reflexology training at a certified training centre in Malaysia (Tim Body Care Training Centre 1403695-D) for 6 months including training and working.

\section{Study outcomes and measures}

There are eight outcomes: two primary outcomes and six secondary outcomes. The two primary outcomes are pain intensity and anxiety level (see table 2 ). Pain intensity is measured with the Present Behavioural Intensity $(\mathrm{PBI})^{71} 72$ and the self-report Visual Analogue Scale (VAS), ${ }^{73-77}$ while anxiety is measure with Anxiety Assessment Scale for Pregnant Women in Labour (AASPWL) ${ }^{78}$

The outcome assessor will ask the pregnant women to pick a colour on an A-4 sized paper that contains six different coloured parts, from no pain (score 1) to most severe pain (score 6) based on her level of pain. ${ }^{7679}$ The researcher selected the VAS questionnaire because it is an acceptable tool and relatively easy to administer to women in labour. Pain intensity will be measured at baseline before the intervention, and multiple times during and after contractions (figure 5A). During the intervention, pain intensity will be measured after the breathing exercise and foot reflexology therapy (after $25 \mathrm{~min}$ from the start of the intervention), followed by another assessment halfway through the massage therapy (after $45 \mathrm{~min}$ ) during and after contraction. Pain intensity will
Table 1 Steps of the intervention

\section{Steps Process}

1. Prepare the equipment.

2. Explain the procedure to the primigravida and advise her to lay on her left side* with a pillow on the side of her stomach.

\section{Breathing exercise intervention for $5 \mathrm{~min}$}

3. Ask the primigravida to perform deep breathing by inhaling slowly through the nose for $2 \mathrm{~s}$ and then consciously release the air by breathing out for another $2 \mathrm{~s}$ during contractions.

4. Rest for $1-3 \mathrm{~s}$, then repeat the same technique for a total of $5 \mathrm{~min}$. Then, proceed to the reflexology as described below.

\section{Reflexology intervention technique for $10 \mathrm{~min}$ on each} foot

5. Put a towel under the right foot and cover the left leg.

6. Apply warm oil over the right foot and roll it left to right five times.

7. Press palms on the Achilles heel and knead the ankle five times.

8. Knead the thumb pads on the central and bottom parts of the heel five times.

9. Knead the foot following the CIUW† shape on the lateral and intermediate aspects of the foot followed by the MST $\neq$ shape five times.

10. Press the wooden reflexology stick on the toes, forefoot, mid-foot and hind-foot five times.

11. Repeat steps 5-11 on the opposite side. Then, proceed to the next lower limbs massage.

\section{Lower limbs massage for $2 \mathrm{~min} 30 \mathrm{~s}$ on each leg}

12. Effleurage massage on the whole, lower flexed leg by using two hands three times.

13. Half effleurage massage from the heel to the popliteal area three times.

14. Palm and thumb kneading on the gastrocnemius muscle over the lateral and medial sides, followed by scooping on the gastrocnemius, each step three times.

15. Thumb kneading on the hamstring muscle over the medial, intermediate and lateral sides three times.

16. Repeat steps $12-17$ on the right leg. Then, proceed to lower back massage.

Lower back massage for $15 \mathrm{~min}$

17. Effleurage massage from the sacrum to the shoulders and deltoids three times.

18. Thumb kneading and pressure over the lateral sides of the lumbar area of the spine three times.

19. Apply fist knuckling motion and thumb kneading on the lower back, side by side, three times. Then, proceed to upper back massage.

\section{Upper back massage for $15 \mathrm{~min}$}

20. Effleurage massage followed by palm kneading from the lumbar region to trapezius laterally three times. 
Table 1 Continued

\section{Steps Process}

21. Thumb kneading over both sides of erector spinae, then draining between the ribs towards the armpit areas three times.

22. Apply squeeze on the deltoid muscle with draining towards the armpit three times.

23. Apply finger kneading on trapezius muscle, followed by fist scooping three times.

24. Finally, press on the neck and shoulder area on both sides three times.

*The left-side position allows maximum blood flow to the placenta, because it applies less pressure from the fetus on the vena cava. ${ }^{70}$ †CIUW shape: C-shape; I-shape, U-shape and W-shape. These shapes indicate the orientation and placement of the palms and knuckles of the therapist.

‡MST shape: M-shape, S-shape and T-shape. These shapes indicate the orientation and placement of the palms and knuckles of the therapist.

be measured for every participating primigravidae in both the intervention and control group. For the control group, pain intensity will be measured first at baseline before the intervention at $6 \mathrm{~cm}$. During the intervention, pain intensity will be measured after $25 \mathrm{~min}$ from the start of the intervention time, followed by another assessment after $45 \mathrm{~min}$, during and after contraction. On completion of the intervention, the measurement will be taken immediately, and twice hourly thereafter during the first stage of labour (figure 5B).

The AASPWL will be used to assess anxiety during labour. The anxiety level will be measured at cervical dilatation of $6 \mathrm{~cm}$, after the completion of the interventions, and twice every $60 \mathrm{~min}$ during the first stage of labour. For the control group, the assessment will be performed when the cervix is at $6 \mathrm{~cm}$, after 1 hour (synchronised to the completion of the intervention in the intervention group), and twice every 60 min during the first stage of labour (figure 5B).

The secondary outcomes measured in the RCT include maternal stress hormones level, maternal V/S, FHR, duration of labour, neonatal Apgar score and maternal satisfaction $^{80}$ (see table 3 ).

The stress hormones level will be measured at baseline, and again one and a half hour after the patient has reached $6 \mathrm{~cm}$ of cervical dilatation (figure 5). Blood samples will again be taken by midwives on duty in the delivery room. This will occur after the BRM intervention in the intervention group (figure $5 \mathrm{~A}$ ), and at the same time in the control group (figure 5B). The research assessors will collect an $8 \mathrm{~mL}$ blood sample in a plain tube, of which $3 \mathrm{~mL}$ is for ACTH, $3 \mathrm{~mL}$ for cortisol and $2 \mathrm{~mL}$ for OT hormones; it will be sent immediately to the MCH laboratory to carefully avoid any haemolysis of the samples. Hormones will be analysed by the sandwich ELISA technique using commercial kits by Cobas e411 Analyzer (HITACHI, USA) for ACTH hormone, and
Abbot Architect I200 Analyzer (Abbott, USA) for Cortisol and OT hormones.

Since cortisol levels follow a diurnal variation or circadian rhythm where the hormone levels peak in the morning and fall at night, and vary in accordance with a number of factors including age, time of day, stress level, sample type, laboratory location and the method used for testing, ${ }^{81}$ we will use a chart from the laboratory to verify the normal cortisol range in the morning, noon, afternoon, evening or night, and compare these ranges to the blood samples taken to determine whether the blood test results of the participants before and after the intervention are high, normal or low.

ACTH and cortisol levels are interrelated. When the cortisol levels are at their peak, ACTH levels generally fall and vice versa. ${ }^{83}$ Hence, it may be understood that ACTH and cortisol have corresponding levels at any given point of time. This provides a relative value for both stress hormones. With regard to OT, the blood sample test for the pregnant woman will be higher if she receives an infusion of OT during intervention. ${ }^{84} 85$ If the events are equal in both groups, we will proceed to the analysis as planned. If the events are many and unequal in both groups, we will either conduct a separate statistical analysis stratified according to the event of OT infusion. If the number of event occurrences is low, we may exclude these participants and analyse the outcome as planned. However, the author will investigate the oxytocin level before and after applying the BRM in addition to childbirth. Oxytocin increases with contraction and hypothetically with and after BRM. The oxytocin will increase in the second stage, inhibit the stress hormones, and will increase after apply the BRM.

Maternal V/S and FHR will be collected twice at $6 \mathrm{~cm}$ cervical dilatation and immediately post-BRM for the intervention group, and the same data will be collected at the same timing for the control groups. The Apgar Scores (taken from the delivery room medical record) and maternal satisfaction will be measured only once at the completion of the childbirth, before the transfer of the mother from the delivery room to the postnatal ward.

\section{Sample size}

The sample size estimation was based on a review of similar literature on pain and anxiety as outcomes, and calculated using $G^{*}$ power free software ${ }^{86}$ We estimated an effect size of 0.6 on anxiety mean score reduction in the intervention group compared with the control, ${ }^{86}$ as this gives a larger required sample size compared with that based on the primary outcome of pain. Thus, with the power of $95 \%$ at $\alpha$ error 0.05 , the required sample size is 128 for the two groups. It is further inflated to 154 to account for a predicted $20 \%$ attrition rate. Therefore, a minimum number of 77 primigravidae will be recruited for each group. 
13 Research Assistants + 1 Principal Investigator

\section{1}

- Recruitment of nursing students who completed a 5-year nursing degree training and awaiting their job posting.

- Assigned to their preferred and suitable roles (coordinators, the outcome assessors, or massage therapists).

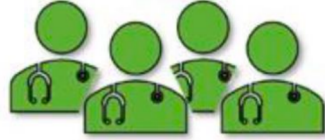

Research coordinators (4 pax.)

- Recruit eligible primigravidae at the antenatal clinic deliver brief health education on labour pain management at the antenatal clinic for 6 weeks.

- Reassess the primigravidae women's consent and eligibility,

- Allocate the women to either the intervention or control group.

- Alert the massage therapists and outcome assessors when the cervical dilatation of the trial participant reaches $6 \mathrm{~cm}$.

- Distribute outcomes assessment record form to the outcome assessors.

- Encode the questionnaire package according to the participant's allocated group.

- Organise the entry sequence for the outcome assessors and the massage therapists to enter the delivery rooms according to the scheduled time.

- One week training as research assistants in their respective roles.

- A pilot study to ensure their competency.

- Written study manual as a reference guide to be provided to all research assistants.

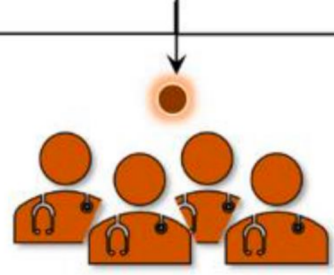

Outcome Assessors (4 pax.)

- Assess and fill-up questionnaires:

1. Present behavioural Intensity (PBI).

2. Visual Analog Scale (VAS).

3. Anxiety Assessment Scale for primigravidae Women in labour (AASPWL),

4. Six Simple Questions (SSQ) for maternal satisfaction.

- Retrieve the maternal vital signs, fetal heart rate, duration of labour, and neonatal Apgar score from the health records.

- Record all the outcomes in the designated form.

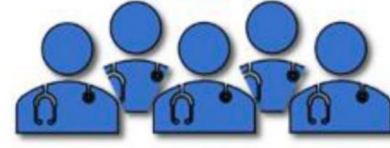

Massage therapists (5 pax.)

- Perform breathing exercise foot reflexology and back massage during labour (BRM).

1. Breathing exercise (5min).

2. Foot Reflexology (10 min on each foot),

3. Back massage (35min).

Sub-divided into two shifts per day to ensure all the eligible and consented primigravidae will be captured

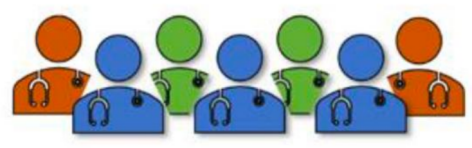

Day Shift (9 am - 9pm)

2 Coordinators, 3 Massage therapists, and 2 Outcome Assessors

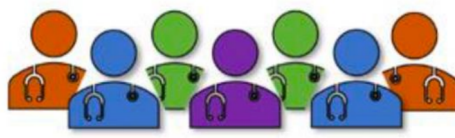

Night Shift (9 pm - 9 am)

2 Coordinators, 2 Massage therapists, 2 Outcome Assessors, and Principal Investigator

Figure 4 Research personnel training and responsibility matrix.

\section{Statistical analysis}

Data will be entered by a blinded enumerator. The database will be checked for accuracy before analysis. The principal investigator has the overall responsibility for the compilation, maintenance and management of the study database. The analysis will be performed using IBM Statistical Package for Social Science V.25.
Descriptive statistical analysis will be performed according to the distribution of the data, using means and SD for data with normal distribution, and median and IQRs for data that are not normally distributed. Normality testing will be conducted for all continuous variables using different methods such as Histogram and $\mathrm{p}-\mathrm{p}$ plot. Categorical variables will be reported in frequencies and percentages. 
Table 2 Summary of primary outcomes and measurement tools

\begin{tabular}{|c|c|c|c|}
\hline Primary outcomes & Tools & Psychometric tests & Method of assessment \\
\hline \multirow[t]{2}{*}{ Pain } & $\mathrm{PBI}$ & $\begin{array}{l}100 \% \text { inter-rater reliability } \\
\text { r coefficient was } 0.45,0.50 \text { and } 0.44 \text { between PBI } \\
\text { and PPI. }{ }^{7172}\end{array}$ & $\begin{array}{l}\text { Assessor-rated, }{ }^{71} \text { five-category } \\
\text { behavioural observation scale }\end{array}$ \\
\hline & VAS & $\begin{array}{l}\text { Moderate correlation }(r=0.54) \text { with the verbal rating } \\
\text { and is considered valuable when mixed with other } \\
\text { tools }{ }^{73} 74 ; 0.97 \text { intraclass correlation coefficient of } \\
24 \text { hours interval test-retest reliability. }\end{array}$ & $\begin{array}{l}\text { Self-reported VAS, }{ }^{76} \text { contains six } \\
\text { different coloured parts anchored } \\
\text { by two extremes of 'no pain' and } \\
\text { excruciating pain to mark on the line } \\
\text { map by primigravidae. }{ }^{77}\end{array}$ \\
\hline Anxiety & AASPWL & $\begin{array}{l}>0.8 \text { concordance test content validity index } \\
\text { Kendall's } W \text { between the opinions of the experts } \\
(\mathrm{W}=0.090 ; p=0.080) \text { with Cronbach's alpha level of } \\
0.77^{78} ; \text { significantly correlated } \\
(r=0.369) \text { with the Beck Anxiety Scale. }{ }^{78}\end{array}$ & $\begin{array}{l}\text { Questionnaire consists of nine } \\
\text { items, }{ }^{78} \text { on a five-point scale: the } \\
\text { higher the mean score, the higher } \\
\text { the anxiety }\end{array}$ \\
\hline
\end{tabular}

AASPWL, Anxiety Assessment Scale for Pregnant Women in Labour; PBI, Present Behavioural Intensity; VAS, Visual Analogue Scale.

The differences between the groups and times level will be analysed using a generalised linear mixed model (GLMM) . GLMM is appropriate where repeated measurements are made on the same statistical units. GLMM will also be used to accommodate non-normal distribution in outcome data. The variables of time in a categorical form, intervention group, group*time interaction and the baseline random part of the model will include a random intercept and an unstructured correlation matrix for the correlation of measurements within pregnant women. The fixed part of the model will include pain score, whereby the difference in pain score at every time point will be tested using a linear contrast. We will take the pain intensity measured with PBI and VAS at 1 hour post intervention as the main coprimary outcomes. This is because the effects of the massage and reflexology will still be observable, and thus the intervention group can be fairly compared with the control group. ${ }^{46-50}$

Any significant baseline imbalances will be adjusted for in the analysis. If necessary, multiple imputations will be conducted for the missing data. A calculated $95 \% \mathrm{CI}$ and two-sided $\alpha$ of 0.05 will be used to test significance. In addition, we will analyse PBI and VAS at the same time points and measure the agreement between PBI and VAS by using the Spearman correlation coefficient and interclass correlation. We will analyse other outcomes using
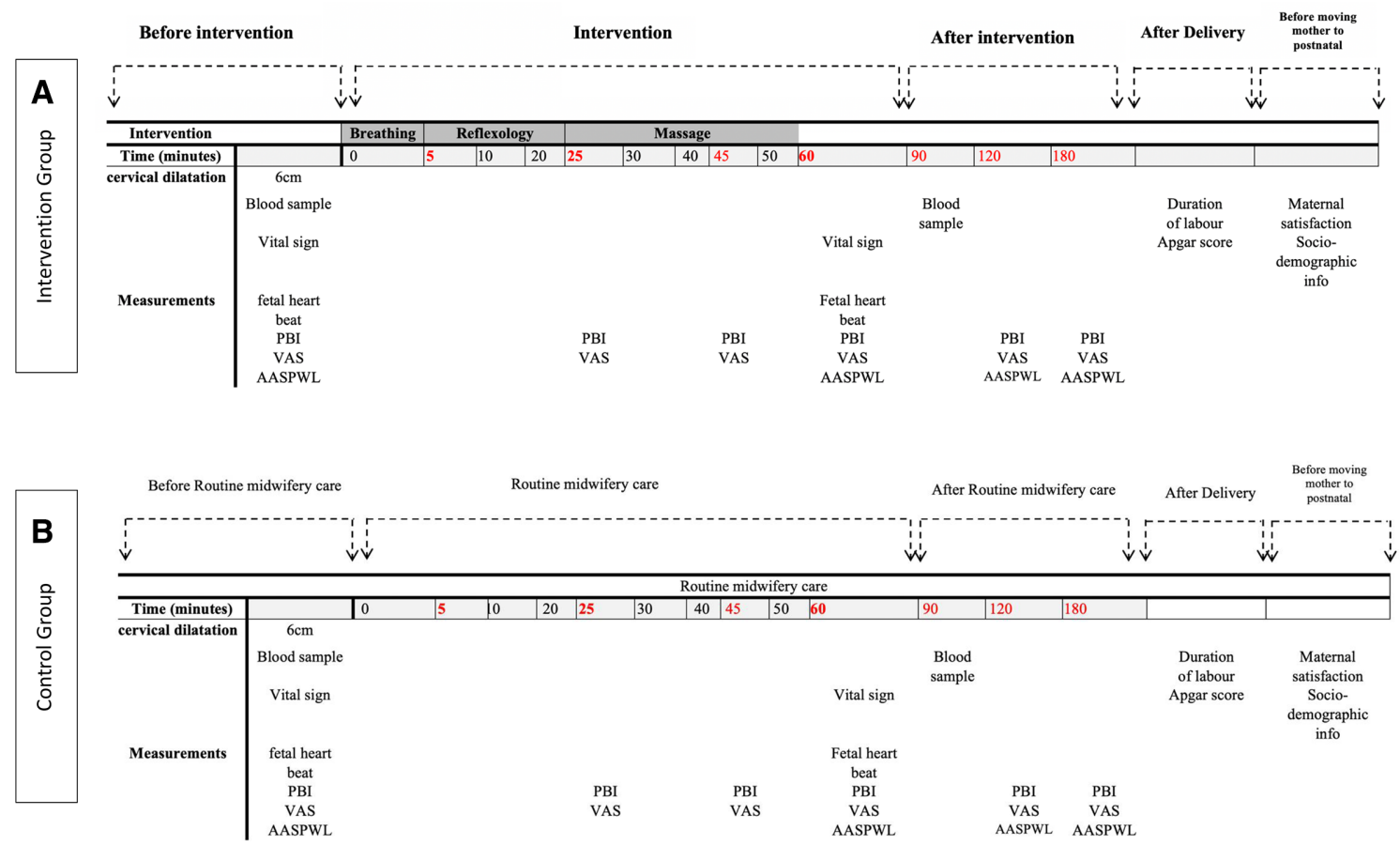

Figure 5 (A) Timeline of outcomes measurement in the intervention group. (B) Timeline of outcomes measurement in the control group. AASPWL, Anxiety Assessment Scale for Pregnant Women in Labour; PBI, Present Behavioural Intensity; VAS, Visual Analogue Scale. 
Table 3 Summary of secondary outcomes and measurement tools

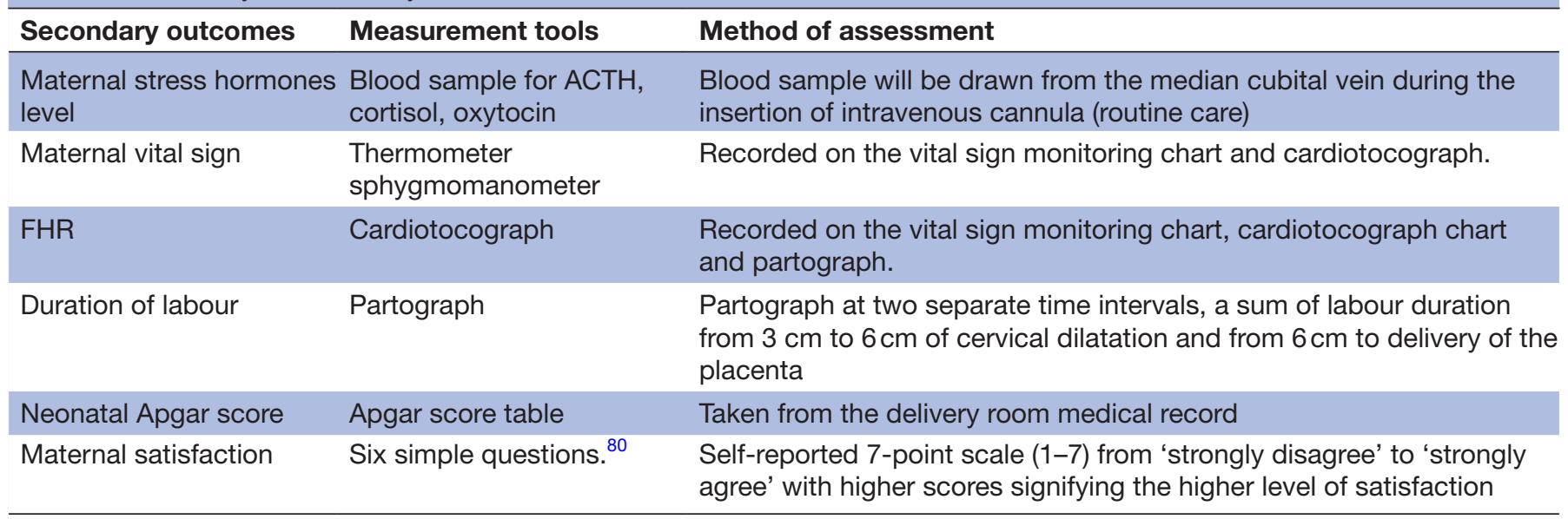

ACTH, adrenocorticotropic hormone; FHR, fetal heart rate.

the same statistical strategy mentioned above. Additionally, we will conduct time series analyses to examine the patterns of change in the outcomes between the two groups and after BRM intervention.

The independent effect(s) of sociodemographic and obstetric characteristics on each primary and secondary outcome at 1 hour post intervention will be analysed using multiple linear regression analyses.

\section{DISCUSSION}

Safe and efficient pain management is important for pregnant women and their families, ${ }^{18}$ and different types of CAM have been shown to be beneficial to reduce or alleviate labour pain. However, evidence is scarce regarding the effects of combined therapies. ${ }^{87}$ Therefore, we designed this trial to study the effects of BRM on labour pain and other psychological and physiological impacts among primigravidae. The study protocol for the RCT is to determine the combined effect of BRM on the intensity of pain and level of anxiety in primigravidae during the first stage of labour. Additional outcomes that will be assessed include stress hormones, maternal V/S, FHR, duration labour, neonatal Apgar score and maternal satisfaction.

In this study, the intervention will be applied only once and only during the first stage of labour even though the first stage of labour among primigravidae takes approximately 8-12hours. By timing the intervention after cervical dilation of $6 \mathrm{~cm}$, the effect of the combined BRM could exert its greatest influences (if any) on the labour experience of the primigravidae and neonatal outcome, because this period is believed to accompany the highest levels of labour pain. ${ }^{88} 89$

We will assess the outcomes using a mixture of subjective and objective tools. For example, pain intensity and anxiety levels are subjective measurements, based on the personal feelings and judgments of the respondents. Duration of labour, neonatal Apgar score and maternal stress hormones level of ACTH, cortisol and oxytocin are objective measurements that will indicate the stress response to the BRM intervention conducted on the primigravidae. This is one of the strengths of our study.

VAS is one of several ways of measuring the effectiveness of BRM, and is a commonly used graphic rating method. ${ }^{70}$ However, VAS might not be the gold standard to measure labour pain, given the inconsistency of its results and its ceiling effect. ${ }^{78}$ Recognising this inadequacy, we will ensure that the participants understand the VAS scoring at admission to the delivery room before they are asked to indicate their pain level later. Labour pain outcome will also be measured via pain intensity assessment using the PBI, ${ }^{74}$ which will be rated by outcome assessors. Multiple measurements will be taken during and after contraction, and before and after the intervention. There will also be other outcomes, related to maternal response to pain, namely anxiety level and maternal stress hormones. ${ }^{91}$

This study has several other limitations. First, the intervention will be performed for 1 hour, during which it may be interrupted by routine medical care such as regular vaginal examinations, V/S measurements and FHR monitoring. However, we believe that this will not reduce the effect of the BRM intervention, because we can start the BRM before or after the labour care routine. Second, the process of labour and birthing is unpredictable even if the participants are at low risk. In certain instances, the process of the intervention might not go well as planned and this may reduce the sample size. Some patients may end up needing a caesarean section, and some may suffer from other obstetric complications during delivery. As a result, we have inflated the sample size accordingly. Third, the results from this study will not be generalisable to multigravidae as we include only primigravidae. Nevertheless, we believe that primigravidae will benefit the most from the intervention as they are likely to experience a higher level of labour pain and a longer duration 
of labour compared with multigravidae. Fourth, placebo effects can influence patient outcomes after (CAM), resulting in high rates of good outcomes, which may be wrongly attributed to specific treatment effects. ${ }^{92}$

We recognise that the expertise and experience level of the reflexologist is an important factor in the quality of treatment provided and this may affect the outcomes of the BRM. The massage therapists and the outcome assessors will be given the appropriate training on the BRM for 1 week by the principal investigator who attended a professional training and was certified. After the training, they will be tested in a pilot study to ensure their competency in performing the BRM. Additional quality control measures for the outcome assessors are planned, as they will be assigned to the control delivery room or the intervention delivery room on the same day. All of the completed assessment forms will be reviewed and kept by the research coordinator in a safe location in the delivery room. Any issues on the form such as blank spaces and extreme values will be immediately clarified and resolved.

In addition to labour pain, this study will assess the anxiety level of pregnant mothers. Unlike labour pain, anxiety level can be affected by individual characteristics, previous life experiences and other environmental causes. ${ }^{93}$ However, we believe that these factors will not play a significant role after effective randomisation.

Apart from the actual labour experience, there are a few other external factors that may affect maternal satisfaction, such as the delivery room services, the health of the baby, the gender of the child, family support and other psychosocial factors. As satisfaction is a multidimensional and complex feeling, it is difficult to measure with a single tool and to narrow it down to only the first stage of labour.

It is understood that a birthing process is a natural event, especially for low-risk women. Thus, the management of labour should be conducted in a supportive manner with minimal or no interferences. This study will provide high-quality evidence about the effects of the combined BRM for labour pain management. These findings will be important for hospitals offerings for expectant mothers in providing a rationale for their decisions about which alternative treatments to offer, to primigravidae and their family members during decision-making about labour pain management.

\section{Ethics approval and consent to participate}

Ethics approval was obtained from the Ethical Committee for Research Involving Human Subjects of the Ministry of Health in the Saudi Arabia (H-02-K-076-0319-109) on 14 April 2019, and from the Ethics Committee for Research Involving Human Subjects (JKEUPM) Universiti Putra Malaysia on 23 October 2019, reference number (JKEUPM-2019-169). Additional administrative approval will be requested from the medical director of the MCH. The participant information sheet for the pregnant women will be also provided. If they are interested and eligible to participate, pregnant women will sign consent forms. Consent form contains purpose of this study, procedures involved in the research preintervention and postintervention. They will inform the potential benefits and risk of the intervention research. Participants will be given an affirmation of confidentiality and protection of the data collection. The results will not be disseminated to the study participants, except if one of the participants would like to know her results, her mobile number will be taken and a message will be sent.

\section{Patient and public involvement}

Patients are involved in the questionnaire's face and content validity testing. Based on feedback from the patients in a pilot study, improvement to the questionnaires' approaches and trial processes will be implemented. Patient preferences were not directly obtained with regard to choosing the BRM intervention; this was based on the principal investigator's practice experience and encounters with pregnant women. However, the patients will be involved in the recruitment to and conduct of the study. They will attend antenatal class and agreement by consent to share in this study. Also, they will answer all questionnaires pre and post the intervention. In addition, they will need to agree to $\mathrm{BRM}$ as the intervention.

\section{Author affiliations}

${ }^{1}$ Department of Family Medicine, Faculty of Medicine and Health Sciences, Universiti Putra Malaysia, Serdang, Malaysia

${ }^{2}$ Department of Nursing, Umm Al-Qura University, Makkah, Saudi Arabia

${ }^{3}$ Department of Nursing \& Rehabilitation, Faculty of Medicine and Health Sciences, Universiti Putra Malaysia, Serdang, Malaysia

Acknowledgements The authors would like to acknowledge the assistance provided by Um-Al-Qura University, the Research Department in the Directorate of Health Affairs, Makkah Region, and Saudi Arabia Culture Mission in Saudi Arabia. Also, they would like to acknowledge Enago for the English language editing services for this report.

Contributors KJB drafted, formulated and submitted the manuscript. BHC and KJB designed the whole study. MHR, AHI and LK contributed to the study designs, and all authors read, revised and approved the research protocol critically for important intellectual content and helped to draft the final manuscript. All authors approved the final manuscript for submission. Authorship eligibility is in accordance with the International Committee of Medical Journal Editors guidelines.

Funding This work was supported by the Umm Al-Qura University and Saudi Arabia Culture Mission. The author (KJB) is a student who owns a Financial Guarantee, which covers the study and research process. The Financial Guarantee will cover all the research's payments.

Disclaimer The funders will not be involved in the study design, data collection, analysis and interpretation of the data and writing of the manuscript.

Competing interests None declared.

Patient and public involvement Patients and/or the public were not involved in the design, or conduct, or reporting, or dissemination plans of this research.

Patient consent for publication Obtained.

Provenance and peer review Not commissioned; externally peer reviewed.

Open access This is an open access article distributed in accordance with the Creative Commons Attribution Non Commercial (CC BY-NC 4.0) license, which permits others to distribute, remix, adapt, build upon this work non-commercially, and license their derivative works on different terms, provided the original work is properly cited, appropriate credit is given, any changes made indicated, and the use is non-commercial. See: http://creativecommons.org/licenses/by-nc/4.0/.

\section{ORCID iDs}

Kamilya Jamel Baljon http://orcid.org/0000-0003-2498-7658

Boon How Chew http://orcid.org/0000-0002-8627-6248 


\section{REFERENCES}

1 Gibson E. Women's expectations and experiences with labour pain in medical and midwifery models of birth in the United States. Women Birth 2014;27:185-9.

2 Beigi NMA, Broumandfar K, Bahadoran P, et al. Women's experience of pain during childbirth. Iran J Nurs Midwifery Res 2010;15:77-82.

3 Devilata T, Swarna S. Effectiveness of pre delivery preparation on anxiety among primigravida mothers at maternal child health centre Tirupati, AP, India. IOSR J Nurs Heal Sci 2015;4:19-24.

4 Aksoy M, Aksoy AN, Dostbil A, et al. The Relationship between Fear of Childbirth and Women's Knowledge about Painless Childbirth. Obstet Gynecol Int 2014;2014:7.

5 Nystedt A, Hildingsson I. Diverse definitions of prolonged labour and its consequences with sometimes subsequent inappropriate treatment. BMC Pregnancy Childbirth 2014;14:233.

6 Hanjani SM, Tourzani ZM, Shoghi M. The effect of foot reflexology on anxiety, pain, and outcomes of the labor in primigravida women. Acta Med Iran 2014;53:507-11.

7 Li W-hong, Zhang H-yu, Ling Y, et al. Effect of prolonged second stage of labor on maternal and neonatal outcomes. Asian Pac J Trop Med 2011;4:409-11.

8 Neal JL, Lamp JM, Buck JS, et al. Outcomes of nulliparous women with spontaneous labor onset admitted to hospitals in Preactive versus active labor. J Midwifery Womens Health 2014;59:28-34.

9 Kjaergaard H, Olsen J, Ottesen B, et al. Incidence and outcomes of dystocia in the active phase of labor in term nulliparous women with spontaneous labor onset. Acta Obstet Gynecol Scand 2009;88:402-7.

10 Lowe NK. A review of factors associated with dystocia and cesarean section in nulliparous women. J Midwifery Womens Health 2007;52:216-28.

11 Shields SG, Ratcliffe SD, Fontaine P, et al. Dystocia in nulliparous women. Am Fam Physician 2007;75:1671-8.

12 Garthus-Niegel S, Knoph C, von Soest T, et al. The role of labor pain and overall birth experience in the development of posttraumatic stress symptoms: a longitudinal cohort study. Birth 2014;41:108-15.

13 Peeler S, Stedmon J, Chung MC, et al. Women's experiences of living with postnatal PTSD. Midwifery 2018;56:70-8.

14 Boryri T, Noori NM, Teimouri A, et al. The perception of primiparous mothers of comfortable resources in labor pain (a qualitative study). Iran J Nurs Midwifery Res 2016;21:239-46.

15 Størksen HT, Garthus-Niegel S, Adams SS, et al. Fear of childbirth and elective caesarean section: a population-based study. BMC Pregnancy Childbirth 2015;15:221.

16 Stoll KH, Hauck YL, Downe S, et al. Preference for cesarean section in young nulligravid women in eight OECD countries and implications for reproductive health education. Reprod Health 2017;14:116.

17 El-Aziz SNA, Mansour SE, Hassan NF. Factors associated with fear of childbirth: It's effect on women's preference for elective cesarean section. J Nurs Educ Pract 2017;7:133-45.

18 Lowe NK. The pain and discomfort of labor and birth. J Obstet Gynecol Neonatal Nurs 1996;25:82-92.

19 Rayburn WF, Zuspan FP. Drug therapy in obstetrics and gynecology. 3rd edn. St. Louis: Mosby, 1992.

20 Ullman R, Smith LA, Burns E, et al. Parenteral opioids for maternal pain relief in labour. Cochrane Database Syst Rev 2010:CD007396.

21 Pereira RR, Kanhai H, Rosendaal F, et al. Parenteral pethidine for labour pain relief and substance use disorder: 20-year follow-up cohort study in offspring. BMJ Open 2012;2:e000719.

22 Smith LA, Burns E, Cuthbert A. Parenteral opioids for maternal pain management in labour. Cochrane Database Syst Rev 2018;6:CD007396.

23 Simkin P. Reducing pain and enhancing progress in labor: a guide to nonpharmacologic methods for maternity caregivers. Birth 1995;22:161-71.

24 Cook A, Wilcox G. Pressuring pain. alternative therapies for labor pain management. AWHONN Lifelines 1997;1:36-41.

25 Jones LV. Non-pharmacological approaches for pain relief during labour can improve maternal satisfaction with childbirth and reduce obstetric interventions. Evid Based Nurs 2015;18:70.

26 Busch V, Magerl W, Kern U, et al. The effect of deep and slow breathing on pain perception, autonomic activity, and mood processing--an experimental study. Pain Med 2012;13:215-28.

27 Thomas ME, Dhiwar MS. Effectiveness of patterned breathing technique in reduction of pain during first stage of labour among Primigravidas. Sinhgad E-Journal Nurs 2011;1:6-8.

28 Kaur K, Rana AK, Gainder S. Effect of video on 'breathing exercises during labour ' on pain perception and duration of labour among primigravida mothers. Nurs Midwifery Res Journa 2013;9:1-9.

29 El-Refaye G, El Nahas E, Ghareeb H. Effect of kinesio taping therapy combined with breathing exercises on childbirth duration and labor pain: a randomized controlled trial. Bull Fac Phys Ther 2016;21:23-31.

30 Chuang L-L, Lin L-C, Cheng P-J, et al. Effects of a relaxation training programme on immediate and prolonged stress responses in women with preterm labour. J Adv Nurs 2012;68:170-80.

31 Akbarzadeh M, Sharif F, Zare N. The reduction of anxiety and improved maternal attachment to fetuses and neonates by relaxation training in primigravida women. Women's Heal Bull 2014;1:e18968.

32 Nattah FM, Abbas WAK. Assessment of level of pain and its relation with breathing exercise in the first stage of labour among Primi mothers at Hilla teaching hospital. Eur J Sci Res 2016;135:121-8.

33 Yuksel H, Cayir Y, Kosan Z, et al. Effectiveness of breathing exercises during the second stage of labor on labor pain and duration: a randomized controlled trial. J Integr Med 2017;15:456-61.

34 Mathew AM, Francis F. Effectiveness of foot reflexology in reduction of labour pain among mothers in labour admitted at PSG Hospital, Coimbatore. Int J Nurs Educ 2016;8:11-15.

35 Cohen M. The effectiveness of massage therapy a summary of evidence-based research. Melbourne: Australian Association of Massage Therapists, 2011.

36 Dolatian1 M, Hasanpour A, Montazeri S, et al. The effect of reflexology on pain intensity and duration of labor on primiparas. Iran Red Crescent Med J 2011;13:475-9.

37 Valiani M, Shiran E, Kianpour M, et al. Reviewing the effect of reflexology on the pain and certain features and outcomes of the labor on the primiparous women. Iran J Nurs Midwifery Res 2010;15:302-10.

38 Baljon K, Romli MH, Ismail $\mathrm{AH}$, et al. The effectiveness of complementary and alternative medicine of massage and reflexology in managing pain and anxiety among primigravidas in labor: a systematic review.

39 Field T, Hernandez-Reif M, Diego M, et al. Cortisol decreases and serotonin and dopamine increase following massage therapy. Int $J$ Neurosci 2005;115:1397-413.

40 Crowe K, von Baeyer C. Predictors of a positive childbirth experience. Birth 1989;16:59-63.

41 Ma X, Yue Z-Q, Gong Z-Q, et al. The effect of diaphragmatic breathing on attention, negative affect and stress in healthy adults. Front Psychol 2017;8:1-12.

42 Bordoni B, Purgol S, Bizzarri A, et al. The influence of breathing on the central nervous system. Cureus 2018;10:1-8.

43 EIFattah $\mathrm{AH}$, Metwaly S, Khedr N. Outcomes of foot reflexology on the pain and certain features of the labor for the primiparous women. Life Sci J 2015;12:206-16.

44 Vigotsky AD, Bruhns RP. The role of descending modulation in manual therapy and its analgesic implications: a narrative review. Pain Res Treat 2015;2015:292805.

45 Kaada B, Torsteinbø O. Increase of plasma beta-endorphins in connective tissue massage. Gen Pharmacol 1989;20:487-9.

46 Tiran D, Chummun $\mathrm{H}$. The physiological basis of reflexology and its use as a potential diagnostic tool. Complement Ther Clin Pract 2005;11:58-64.

47 Embong NH, Soh YC, Ming LC, et al. Revisiting reflexology: concept evidence, current practice, and practitioner training. J Tradit Complement Med 2015;5:197-206.

48 Levy I, Attias S, Stern Lavee T, et al. The effectiveness of foot reflexology in reducing anxiety and duration of labor in primiparas: an open-label randomized controlled trial. Complement Ther Clin Pract 2020;38:101085

49 Yilar Erkek Z, Aktas S. The effect of foot reflexology on the anxiety levels of women in labor. J Altern Complement Med 2018;24:352-60.

50 Tappan FM, Benjamin PJ. Tappan's handbook for healing massage techniques. Classic, holistic, and emerging methods. 3rd edn. Stamford, US: Appleton \& Lange, 1998.

51 Taheri H, Naseri-Salahshour V, Abedi A, et al. Comparing the effect of foot and hand reflexology on pain severity after appendectomy: a randomized clinical trial. Iran J Nurs Midwifery Res 2019;24:451-6.

52 Tiran D, Evans M. Theoretical background to structural reflex zone therapy. In: Tiran D, ed. Reflexology in pregnancy and childbirth. London: Elsevier, 2010: 1-22.

53 Poole H, Glenn S, Murphy P. A randomised controlled study of reflexology for the management of chronic low back pain. Eur J Pain 2007;11:878-87.

54 Keet L. The reflexology bible. 1st edn. London: Octopus publishing group, 2008.

55 Roudaut Y, Lonigro A, Coste B, et al. Touch sense: functional organization and molecular determinants of mechanosensitive receptors. Channe/s 2012;6:234-45.

56 Goats GC. Massage--the scientific basis of an ancient art: Part 2. Physiological and therapeutic effects. Br J Sports Med 1994;28:153-6. 
57 Basbaum Al, Fields HL. Endogenous pain control mechanisms: review and hypothesis. Ann Neurol 1978;4:451-62.

58 Weerapong P, Hume PA, Kolt GS. The mechanisms of massage and effects on performance, muscle recovery and injury prevention. Sports Med 2005;35:235-56.

59 Morhenn V, Beavin LE, Zak PJ. Massage increases oxytocin and reduces adrenocorticotropin hormone in humans. Altern Ther Health Med 2012;18:11-18.

60 Li Q, Becker B, Wernicke J, et al. Foot massage evokes oxytocin release and activation of orbitofrontal cortex and superior temporal sulcus. Psychoneuroendocrinology 2019;101:193-203.

61 Bell AF, Erickson EN, Carter CS. Beyond labor: the role of natural and synthetic oxytocin in the transition to motherhood. J Midwifery Womens Health 2014;59:35-42.

$62 \mathrm{Hu}$ J, Brettle A, Jiang Z, et al. A systematic review and meta-analysis of the effect of massage therapy in pain relief during labor. $J$ Nurs 2017:6:1-14.

63 Huntley AL, Coon JT, Ernst E. Complementary and alternative medicine for labor pain: a systematic review. Am J Obstet Gynecol 2004;191:36-44.

64 Ranjbaran M, Khorsandi M, Matourypour P, et al. Effect of massage therapy on labor pain reduction in primiparous women: a systematic review and meta-analysis of randomized controlled clinical trials in Iran. Iran J Nurs Midwifery Res 2017;22:257-61.

65 Smith CA, Levett KM, Collins CT, et al. Massage, reflexology and other manual methods for pain management in labour. Cochrane Database Syst Rev 2018;3:CD009290.

66 Kirkham M. The Midwife-Mother relationship. 2nd edn. Hampshire: Red Globe Press, 2000.

67 Levett KM, Smith CA, Bensoussan A, et al. The Complementary Therapies for Labour and Birth Study making sense of labour and birth - Experiences of women, partners and midwives of a complementary medicine antenatal education course. Midwifery 2016;40:124-31.

68 Ministry of Health Saudi Arabia. Maternity and children Hospital Makkah, 2013. Available: http://www.testonserver.com/mch/ [Accessed 27 Nov 2018].

69 Altaweli RF, McCourt C, Baron M. Childbirth care practices in public sector facilities in Jeddah, Saudi Arabia: a descriptive study. Midwifery 2014;30:899-909.

70 Farine D, Seaward PG. When it comes to pregnant women sleeping, is left right? J Obstet Gynaecol Can 2007;29:841-2.

71 Bonnel AM, Boureau F. Labor pain assessment: validity of a behavioral index. Pain 1985;22:81-90.

72 Chang M-Y, Wang S-Y, Chen C-H. Effects of massage on pain and anxiety during labour: a randomized controlled trial in Taiwan. $J$ Adv Nurs 2002;38:68-73.

73 Mårtensson L, Bergh I. Effect of treatment for labor pain : Verbal reports versus visual analogue scale scores - A prospective randomized study. Int J Nurs Midwifery 2011;3:43-7.

74 Bahammam MA, Hassan MH. Validity and reliability of an Arabic version of the modified dental anxiety scale in Saudi adults. Saudi Med J 2014;35:1384.

75 Hawker GA, Mian S, Kendzerska T, et al. Measures of adult pain: visual analog scale for pain (vas pain), numeric rating scale for pain (nrs pain), mcgill pain questionnaire (mpq), short-form mcgill pain questionnaire (sf-mpq), chronic pain grade scale (cpgs), short form-36 bodily pain scale (sf-36 bps), and measure of intermittent and constant osteoarthritis pain (icoap). Arthritis Care Res 2011;63:S240-52.

76 Taghinejad H, Delpisheh A, Suhrabi Z. Comparison between massage and music therapies to relieve the severity of labor pain. Womens Health 2010;6:377-81.

77 Jensen MP, Karoly P, Braver S. The Measurement of Clinical Pain Intensity : a Comparison of Six Methods. Pain Res Manag 1986;27:117-26.

78 Durat G, Çulhacik GD, Doğu Özlem, et al. The development of an anxiety assessment scale for pregnant women in labor. Saudi Med $J$ 2018:39:609-14.

79 Wei CK, Leng CY, Siew Lin SK. The use of the visual analogue scale for the assessment of labour pain: a systematic review. JBI Libr Syst Rev 2010;8:972-1015.

80 Harvey S, Rach D, Stainton MC, et al. Evaluation of satisfaction with midwifery care. Midwifery 2002;18:260-7.

81 Bublitz MH, Bourjeily G, D'Angelo C, et al. Maternal sleep quality and diurnal cortisol regulation over pregnancy. Behav Sleep Med 2018:16:282-93.

82 Larsson CA, Gullberg B, Råstam L, et al. Salivary cortisol differs with age and sex and shows inverse associations with WHR in Swedish women: a cross-sectional study. BMC Endocr Disord 2009;9:16.

83 Sundaram L, Swaminathan S, Ranjakumar T, et al. Intraocular pressure variation in pregnancy: a prospective study. Int $J$ Reprod Contracept Obstet Gynecol 2017;5:1406-9.

84 Romano A, Tempesta B, Micioni Di Bonaventura MV, et al. From autism to eating disorders and more: the role of oxytocin in neuropsychiatric disorders. Front Neurosci 2015;9:497.

85 Uvnäs-Moberg K, Ekström-Bergström A, Berg M, et al. Maternal plasma levels of oxytocin during physiological childbirth - a systematic review with implications for uterine contractions and central actions of oxytocin. BMC Pregnancy Childbirth 2019;19:285

86 Faul F, Erdfelder E, Lang A-G, et al. G*Power 3: a flexible statistical power analysis program for the social, behavioral, and biomedical sciences. Behav Res Methods 2007;39:175-91.

87 Snyder M, Wieland J. Complementary and alternative therapies: what is their place in the management of chronic pain? Nurs Clin North Am 2003;38:495-508.

88 Sekhavat L, Behdad S. The effects of meperidine analgesia during labor on fetal heart rate. Int J Biomed Sci 2009;5:59.

89 Reynolds $F$. The effects of maternal labour analgesia on the fetus. Best Pract Res Clin Obstet Gynaecol 2010;24:289-302.

90 Whitburn LY, Jones LE, Davey M-A, et al. The nature of labour pain: an updated review of the literature. Women Birth 2019;32:28-38.

91 Woo AKM. Depression and anxiety in pain. Rev Pain 2010;4:8-12.

92 Turner JA, Deyo RA, Loeser JD, et al. The importance of placebo effects in pain treatment and research. JAMA 1994;271:1609-14.

93 Brook CA, Schmidt LA. Social anxiety disorder: a review of environmental risk factors. Neuropsychiatr Dis Treat 2008;4:123-43. 